\title{
Mammalian target of the rapamycin pathway is involved in non-alcoholic fatty liver disease
}

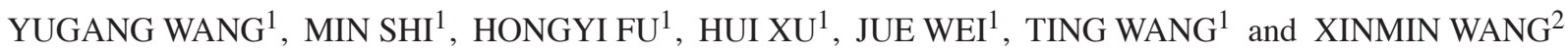 \\ ${ }^{1}$ Department of Gastroenterology, Shanghai Changning Central Hospital, Shanghai 200336; \\ ${ }^{2}$ Department of Gastroenterology, Tongji Hospital of Tongji University, Shanghai 200065, P.R. China
}

Received July 12, 2010; Accepted September 6, 2010

DOI: $10.3892 / \mathrm{mmr} .2010 .365$

\begin{abstract}
Non-alcoholic fatty liver disease (NAFLD) is a common liver disease associated with an increased risk of type 2 diabetes and cardiovascular disease. Many factors may contribute to NAFLD development and progression, but the exact mechanisms are still not fully understood. In this study, Sprague-Dawley rats were fed either a standard diet (control group), a high-fat diet for 8 weeks (the HFD-8 group) or a high-fat diet for 16 weeks (the HFD-16 group). The HFD animals showed high levels of aspartate aminotransferase (AST), alanine aminotransferase (ALT) and insulin resistance index (Homa-IR). Mild and severe steatosis was found in both the HFD-8 and HFD-16 groups, respectively. Compared with the controls, mRNA levels of mTOR, S6K1, IL-1 $\alpha$, IL-6 and TNF $\alpha$ were significantly increased in the HFD-8 and HFD-16 groups. IRS-1 mRNA was significantly increased in the HFD-8 group, but not in the HFD-16 group. The protein levels of mTOR, pmTOR(Ser2448), S6K1, pIRS-1(Ser307), IL- $1 \alpha$ and IL- 6 were significantly increased in the HFD- 8 and HFD-16 groups. The protein levels of pmTOR(Ser2448) and IL-1 $\alpha$ were significantly higher in the HFD-16 group compared to those in the HFD-8 group. However, the protein expression level of mTOR did not differ significantly between the HFD-8 and HFD-16 groups. The pIRS-1(Tyr102) level was significantly lower in both the HFD-8 and HFD-16 groups when compared to that in the control group, and the pIRS-1(Tyr102) level was significantly lower in the HFD-16 group compared to that of the HFD-8 group. pmTOR(Ser2448) was positively correlated with the TNF $\alpha$ mRNA level, and pIRS-1(Ser307) was positively correlated with pmTOR(Ser2448), TNF $\alpha$, S6K1 and mTOR. pIRS-1(Tyr102) was negatively correlated with pmTOR(Ser2448), TNF $\alpha$, S6K1 and mTOR. These data indicate that mTOR contributes to insulin resistance and chronic liver inflammation, and may play an important role in the development and progression of NAFLD.
\end{abstract}

Correspondence to: Dr Xinmin Wang, Department of Gastroenterology, Tongji Hospital of Tongji University, Shanghai 200065, P.R. China

E-mail: xm.wang2010@yahoo.com.cn

Key words: liver disease, rapamycin, rat model, high-fat diet

\section{Introduction}

Non-alcoholic fatty liver disease (NAFLD) affects between 10 and $24 \%$ of the general population in many countries and has become an important public health concern (1). NAFLD is associated with type 2 diabetes mellitus, metabolic syndrome and coronary heart disease (2). However, the pathogenesis of NAFLD is not yet fully understood. Insulin resistance (IR) is the most reproducible factor in the development of NAFLD (3).

Mammalian target of rapamycin (mTOR) is involved in the occurrence of IR and the transmission of insulin signals $(4,5)$. mTOR is a highly conserved serine/threonine kinase that controls cell growth and metabolism in response to nutrients, growth factors, cellular energy and stress (6). Thus, it is hypothesized that mTOR is also involved in the development of NAFLD. Recent studies have revealed that ribosomal protein S6 kinase 1, S6K1, which is an effector of mTOR, is sensitive to both insulin and nutrients. Insulin receptor substrate-1 (IRS-1) is a signaling protein which couples the insulin receptor to the phosphoinositide-3-kinase (PI3K) signaling cascade (7). Serine phosphorylation of IRS-1 disassociates coupling of IRS-1 signal transduction to PI3K and results in IR.

In the present study, a high-fat diet-induced rat NAFLD model was used to investigate the relationship between mTOR and S6K1, IRS-1 and other inflammatory cytokines, including IL-1 $\alpha$, IL- 6 and TNF $\alpha$.

\section{Materials and methods}

Reagents. MMLV reverse transcriptase, TRIzol and SYBR Green I were purchased from Invitrogen (Carlsbad, CA). The Prime Script ${ }^{\mathrm{TM}}$ RT reagent kit was from Takara (Dalian, China). The Pierce ${ }^{\circledR}$ BCA Protein Assay kit and Super Signal ${ }^{\circledR}$ West Femto Maximum Sensitivity substrate were from Thermo Scientific (Waltham, MA). ECL Plus Western blotting detection reagents were from GE Healthcare (Piscataway, NJ). Phospho-mTOR (Ser2448), phospho-IRS-1 (Ser307) and phospho-tyrosine mouse mAb (pTyr102) were from Cell Signaling (Beverly, MA). Ribosomal protein S6 kinase (P70-S6K; RPS6KB1) rabbit polyclonal antibody was from Bionity (Deutschland, Germany). Monoclonal anti-GAPDH antibody was from Sigma (St. Louis, MO). IL-1 
Table I. Primer sequences.

\begin{tabular}{lllll}
\hline Primers & \multicolumn{1}{c}{ Forward primer } & \multicolumn{1}{c}{ Reverse primer } & $\begin{array}{c}\text { Size } \\
\text { (bp) }\end{array}$ & $\begin{array}{c}\text { NCBI reference } \\
\text { sequence }\end{array}$ \\
\hline IRS-1 & AGCTATGCTGACATGCGGACA & CGGCCCCTTGAGGTGTAA & 124 & NM_012969.1 \\
mTOR & CTTATGCCAACCTCCTAGCTG & GTTAAGTCCAAACCCGTAT & 165 & NM_019906.1 \\
S6K1 & GCACCTGCGGATGAATCTAT & TTTTCCTCCATCGAAAGCAT & 165 & NM_031985.1 \\
IL1a & CTCAAACTTGCTTTATTTAATATGA & TAACTGTGACAACCACATCCC & 167 & NM_017019.1 \\
IL6 & TGGAGTCACAGAAGGAGTGGCTAAG & TCTGACCACAGTGAGGAATGTCCAC & 154 & NM_012589.1 \\
TNF $\alpha$ & CACCCACACCGTCAGCCGATT & AGTAGACCTGCCCGGACTC & 227 & X66539.1 \\
GAPDH & TCCTGCACCACCAACTGCTTAGCC & TAGCCCAGGATGCCCTTTAGTGGG & 377 & \\
\hline
\end{tabular}

antibody was from AbD Serotec (Kidlington, UK), and IL-6 antibody was from Santa Cruz (Santa Cruz, CA). Taq polymerase and random primers were from Sangon (Shanghai, China). RNAase inhibitor, dNTP, M-MLV and TEMED were from Promega (Madison, WI). All of the other chemicals used were analytical reagents.

Animal model and groups. Male Sprague-Dawley (SD) rats, weighing 150-180 g, were purchased from Shanghai SLAC Laboratory Animals Co., Ltd. (Shanghai, China) and were housed under standardized conditions $\left(18-23^{\circ} \mathrm{C}\right)$, with 12 -h light/12-h darkness, $60 \%$ humidity and free access to food and tap water. The animals were randomly divided into three groups ( $n=30 /$ group): the control group rats were fed a standard diet; the HFD-8 group received a high-fat diet (standard diet plus 10\% lard, 5\% yolk and 2\% cholesterol) for 8 weeks, and the HFD-16 group recieved a high-fat diet for 16 weeks. All of the studies were performed with the approval of the Experimental Animal Committee of Tongji University, Shanghai, China. After the rats were sacrificed under deep anesthesia, the liver was promptly excised and removed. One portion of the liver was fixed in $10 \%$ formalin, and the remaining liver was stored in liquid nitrogen.

Histological examinations. The liver samples were processed in a series of stages including alcohol dehydration, transparency by xylene, soaking in organic solution and longitudinal embedding in paraffin. The paraffin blocks were cut into $4-\mu \mathrm{m}$ tissue sections for staining with hematoxylin and eosin (H\&E) under a light microscope (Olympus, Tokyo, Japan).

Biochemical assays. One day before sacrifice, the animals were fasted. Before the animals were sacrificed, peripheral blood was harvested from the tail veins, and the serum was harvested by centrifugation. AST, ALT and fasting blood glucose (FBG) were measured using an auto-analyzer (Beckman LX-20, Palo Alto, CA). Rat insulin levels (FINS) were detected with the use of the Rat Insulin RIA kit (Linco Research Inc., St. Charles, MO) according to the manufacturer's instructions. HOMA-IR was calculated by using the following equation: HOMA-IR $=($ FBG $x$ FINS $) / 22.5$.

Real-time PCR. For real-time PCR, RNA samples were extracted from the liver tissues using TRIzol according to the manufacturer's instructions (Invitrogen). cDNA was immediately reverse transcribed using $2 \mu \mathrm{g}$ RNA. Real-time PCR was undertaken using the SYBR Green method conducted on the 7300 Real-time PCR system (ABI, Foster City, CA) according to the manufacturer's instructions. The primers were designed using Primer Express ${ }^{\mathrm{TM}} 1.5$ software (ABI) on the basis of sequence data obtained from the NCBI database and were synthesized by Sangon (Table I). The following cycling conditions were used: $95^{\circ} \mathrm{C}$ for $30 \mathrm{sec}$ followed by 42 cycles at $95^{\circ} \mathrm{C}$ for $5 \mathrm{sec}$ and $56^{\circ} \mathrm{C}$ for $30 \mathrm{sec}$. Real-time PCR assays were conducted in triplicate for each sample. The amount of target gene expression was calculated using the respective standard curves, and the quantitative expression was normalized using rat GAPDH.

Western blot analysis. Liver samples were homogenized in a tissue lysis buffer (50 mM Tris-HCl, pH 8.0, $150 \mathrm{mM} \mathrm{NaCl}, 1 \%$ Nonidet P-40) on ice and centrifuged at 3,000 x g for $15 \mathrm{~min}$ at $4^{\circ} \mathrm{C}$ to remove nuclear and particulate matter. The supernatants were collected and centrifuged at 40,000 x g for $30 \mathrm{~min}$ at $4^{\circ} \mathrm{C}$, and the remaining pellet was resuspended in $1 \mathrm{ml}$ of lysis buffer. The amount of total protein in the samples was determined using the Lowry method. Protein samples $(50 \mu \mathrm{g})$ were resolved on a 12\% SDS-PAGE and then electroblotted on PVDF membranes (Bio-Rad). The membranes were incubated with antibodies of mTOR, pmTOR(Ser2448), S6K1, pIRS-1 (Ser307), pIRS-1(Tyr102), IL-1 $\alpha$ or IL-6 (1:1000) overnight at $4^{\circ} \mathrm{C}$, followed by incubation with horseradish peroxidaseconjugated anti-rabbit antibody (1:5000) for $2 \mathrm{~h}$; ECL was used to visualize the bands. The expression of GADPH was used as an internal control to normalize the expression of the other proteins. The bands were quantified using a GIS-2008 image-processing system (Shanghai Tianneng Tech, Shanghai, China).

Statistical analysis. All values were expressed as the mean \pm SD. A statistical package SPSS11.0 (SPSS Inc., Chicago, IL, USA) was used for all analyses. A one-way ANOVA was used to analyze the mean of each group. The least significant difference (LSD) method was used to evaluate the difference between the means of the groups. Pearson's correlation was used to investigate the possible correlations between variables. A value of $\mathrm{P}<0.05$ was considered statistically significant. 
Table II. Changes in biochemical indices.

\begin{tabular}{lllllll}
\hline Group & No. & ALT $(\mathrm{U} / \mathrm{l})$ & AST $(\mathrm{U} / \mathrm{l})$ & FBG $(\mathrm{mmol} / \mathrm{l})$ & FINS (mU/l) & HOMA-IR \\
\hline Control & 10 & $53.55 \pm 11.12$ & $178.21 \pm 9.93$ & $5.53 \pm 0.95$ & $15.33 \pm 3.38$ & $3.86 \pm 1.35$ \\
HFD-8 & 10 & $66.49 \pm 10.15^{\mathrm{d}}$ & $198.67 \pm 12.12^{\mathrm{a}}$ & $5.68 \pm 0.93$ & $22.32 \pm 4.37^{\mathrm{a}}$ & $5.79 \pm 2.04^{\mathrm{d}}$ \\
HFD-16 & 10 & $85.12 \pm 11.41^{\mathrm{a}, \mathrm{b}}$ & $223.51 \pm 21.98^{\mathrm{a}, \mathrm{b}}$ & $6.11 \pm 1.24$ & $28.17 \pm 3.96^{\mathrm{a}, \mathrm{b}}$ & $7.77 \pm 2.42^{\mathrm{a}, \mathrm{c}}$ \\
\hline
\end{tabular}

Index values are represented as the mean $\pm \mathrm{SD}$. ${ }^{\mathrm{P}}<0.01$ vs. control group; ${ }^{\mathrm{b}} \mathrm{P}<0.01$ vs. HFD-8 group; ${ }^{\mathrm{P}}<0.05$ vs. HFD-8 group; ${ }^{\mathrm{d}} \mathrm{P}<0.05$ vs control group. ALT, alanine aminotransferase; AST, aspartate aminotransferase; FBG, fasting blood glucose; FINS, fasting serum insulin; Homa-IR, insulin resistance index.

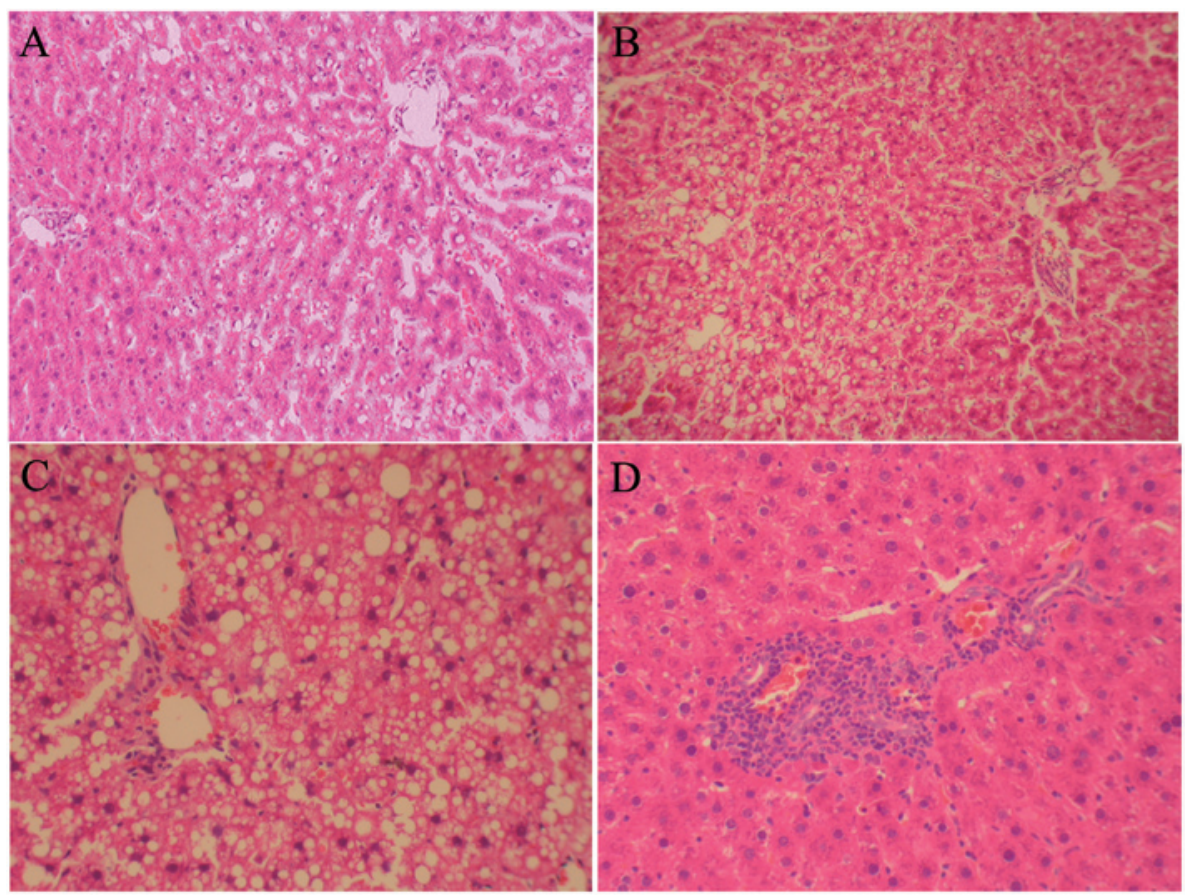

Figure 1. Histopathology of rats fed a high-fat diet for 8 or 16 weeks. Sections were stained with H\&E. (A) Mild steatosis, fat present in 30-50\% of hepatocytes; (B) moderate steatosis, fat present in $50-75 \%$ of hepatocytes; (C) severe steatosis, fat present in $>75 \%$ of hepatocytes; and (D) a large number of inflammatory cells infiltrated at the portal area.

\section{Results}

Effect of a high-fat diet on biochemical indices. As shown in Table II, ALT and AST levels were significantly higher in the HFD-8 and HFD-16 groups compared to the control group $(\mathrm{P}<0.01)$, and ALT and AST levels were significantly higher in the HFD-16 group than those in the HFD-8 group. This indicates that a high-fat diet increases ALT and AST levels in a time-dependent manner. Although there was no significant difference in FBG between the HDT groups and the control group ( $\mathrm{P}>0.05)$, FINS and HOMA-IR in the HFD groups were significantly higher than the levels in the control group $(\mathrm{P}<0.05)$. FINS and HOMA-IR were significantly higher in the HFD-16 group than those in the HFD-8 group $(\mathrm{P}<0.05)$, indicating that a high-fat diet increases FINS and HOMA-IR levels in a time-dependent manner.

Histopathological lesions. Administration of a high-fat diet for eight weeks caused mild (fat present in 30-50\% of hepa- tocytes) or moderate (fat present in $50-75 \%$ of hepatocytes) steatosis (Fig. 1A and B). Administration of a high-fat diet for 16 weeks caused severe steatosis (fat present in more than $75 \%$ of hepatocytes) (Fig. 1C). Large numbers of inflammatory cells, such as lymphocytes and neutrophils, were found to have infiltrated the portal area. Some inflammatory cells had destroyed the limiting plate and had infiltrated into the liver lobules and around the hepatocytes (Fig. 1D).

Changes in mTOR, IRS-1, S6K1, IL-1 $\alpha, I L-6$ and TNF $\alpha$ mRNA levels. Compared to the control groups, mTOR, S6K1, IL-1 $\alpha$, IL-6, and TNF $\alpha$ mRNA levels were significantly increased $(\mathrm{P}<0.01)$ in the HFD-8 and HFD-16 groups. The IRS-1 mRNA level was significantly higher in the HFD-8 group comapred with that in the control group, but no differences were observed between the HFD-16 group and the control group ( $\mathrm{P}>0.05$; Fig. 2). These data were in accordance with the histopathological findings. Along with the prolonging of the high-fat diet, the accumulation and infiltration of inflamma- 
a

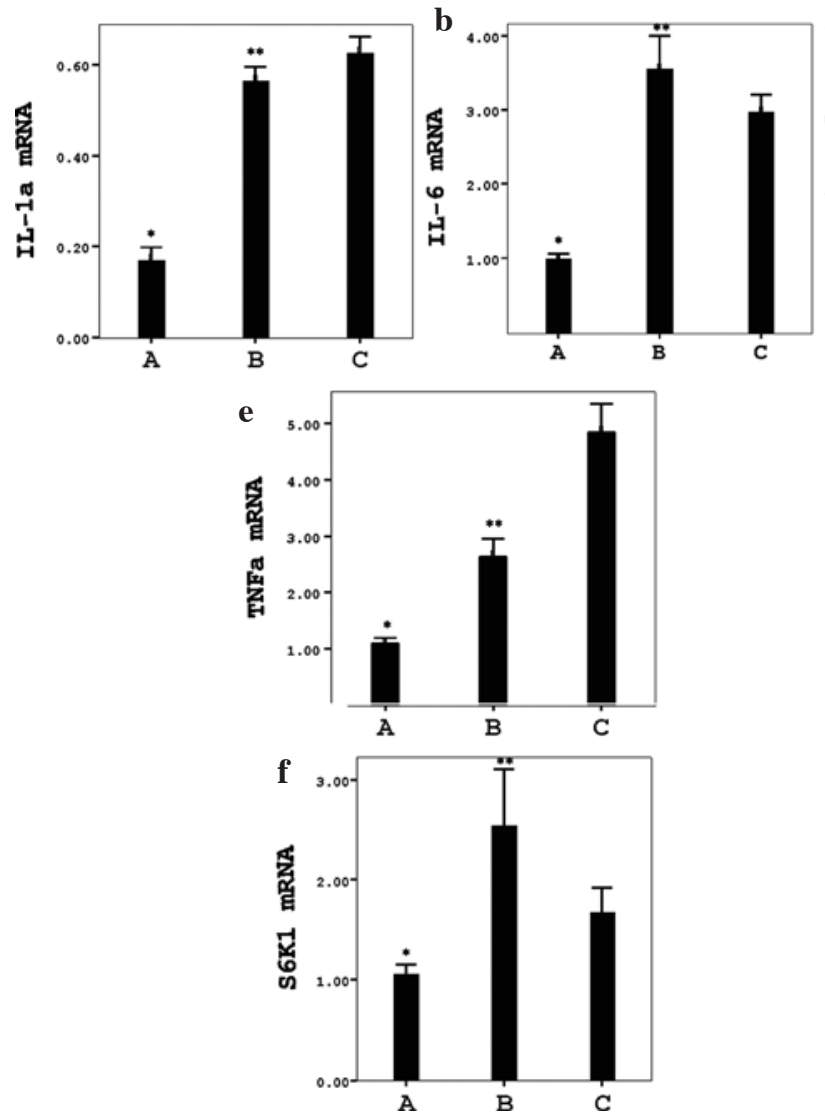

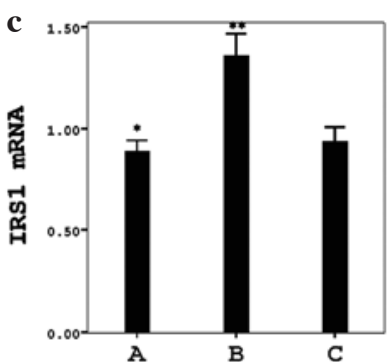
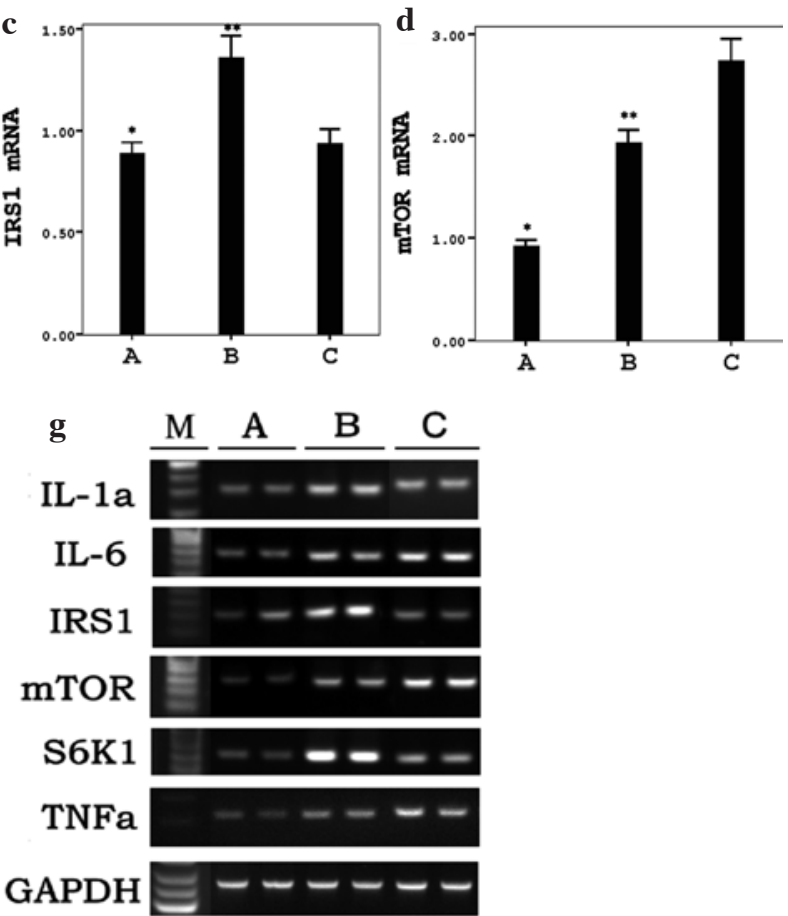

Figure 2. mRNA level of IL-1 $\alpha$ (a), IL-6 (b), IRS-1 (c), mTOR (d), TNF $\alpha$ (e) and S6K1 (f). GADPH was used as an internal control. Values are the means \pm SD. A, control group; B, HFD-8 group; C, HFD-16 group. *A compared with $\mathrm{B}$ or $\mathrm{C}, \mathrm{P}<0.05$. ${ }^{*} \mathrm{~B}$ compared with $\mathrm{C}$, $\mathrm{P}<0.05$. A representative real-time $\mathrm{PCR}$ image is shown $(\mathrm{g})$.

tory cells in the liver increased. Therefore, the mTOR/S6K1 signaling pathway appears to be mediated by the biological effects of a high-fat diet.

Changes in mTOR, pmTOR(Ser2448), S6K1, pIRS-1 (Ser307), pIRS-1(Tyr102), IL-1 $\alpha$ and IL-6 protein levels. As shown in Fig. 3, compared with the control group, mTOR, pmTOR(Ser2448), S6K1, pIRS-1(Ser307), IL-1 $\alpha$ and IL-6 protein expression levels were significantly increased $(\mathrm{P}<0.01)$ in the HFD-8 and HFD-16 groups. pmTOR(Ser2448) and IL-1 $\alpha$ protein expression levels were significantly higher in the HFD-16 group than those in the HFD-8 group. The pIRS-1(Tyr102) protein expression level was significantly lower in both the HFD-8 and HFD-16 groups compared to that of the control group $(\mathrm{P}<0.01)$, and the pIRS-1(Tyr102) protein expression level was significantly lower in the HFD-16 group compared to that of the HFD-8 group $(\mathrm{P}<0.05)$, but no significant differences in mTOR protein expression were observed between the HFD-16 and the HFD-8 group ( $\mathrm{P}>0.05)$. These data indicate that a high-fat diet leads to an increase in the phosphorylation of mTOR and an increase in S6K1 expression, resulting in the activation of the mTOR/S6K1 signaling pathway. A high-fat diet decreased the phosphorylation of pIRS-1, indicating that a high-fat diet decreases the sensitivity to insulin. The effect of a high-fat diet on mTOR and pIRS occurred in a timedependent manner.
Correlation analysis. In order to understand the relationships among the mTOR/S6K1 signaling pathway, IRS-1 and inflammatory cytokines, a Pearson's correlation analysis was performed. We found that pmTOR(Ser2448) was positively correlated with TNF $\alpha$ mRNA ( $\mathrm{r}=0.892, \mathrm{P}<0.001)$. pIRS-1 (Ser307) was positively correlated with pmTOR(Ser2448) $(\mathrm{r}=0.752, \mathrm{P}<0.001), \mathrm{TNF} \alpha$ mRNA ( $\mathrm{r}=0.604, \mathrm{P}=0.019), \mathrm{S} 6 \mathrm{~K} 1$ $(\mathrm{r}=0.530, \mathrm{P}<0.001)$ and mTOR $(\mathrm{r}=0.904, \mathrm{P}<0.001)$. pIRS-1 (Tyr102) was negatively correlated with pmTOR(Ser2448) $(\mathrm{r}=-0.888, \mathrm{P}<0.001), \mathrm{TNF} \alpha \mathrm{mRNA}(\mathrm{r}=-0.828, \mathrm{P}<0.001), \mathrm{S} 6 \mathrm{~K} 1$ $(r=-0.631, P<0.001)$ and mTOR $(r=-0.904, P<0.001)$.

\section{Discussion}

In the present study, a high-fat diet-induced NAFLD rat model was used to investigate the role of mTOR in the expression of inflammatory cytokines, insulin resistance and steatosis. In recent years, there has been an increase in the incidence of NAFLD $(8,9)$. The pathogenesis of NAFLD is well-described and involves both genetic susceptibility and the development of a multifactorial metabolic disorder. The pathophysiological basis of the disease consists largely of insulin resistance and oxidative stress. However, to date, there is no complete theory that fully explains all the clinical signs of NAFLD $(10,11)$. Currently, IR with hepatocyte steatosis $(12,13)$ and inflammatory attacks, caused by oxidative stress and activation of TNF $\alpha$ and $\mathrm{NF}-\kappa \mathrm{B}$, are widely recognized as significant factors that 

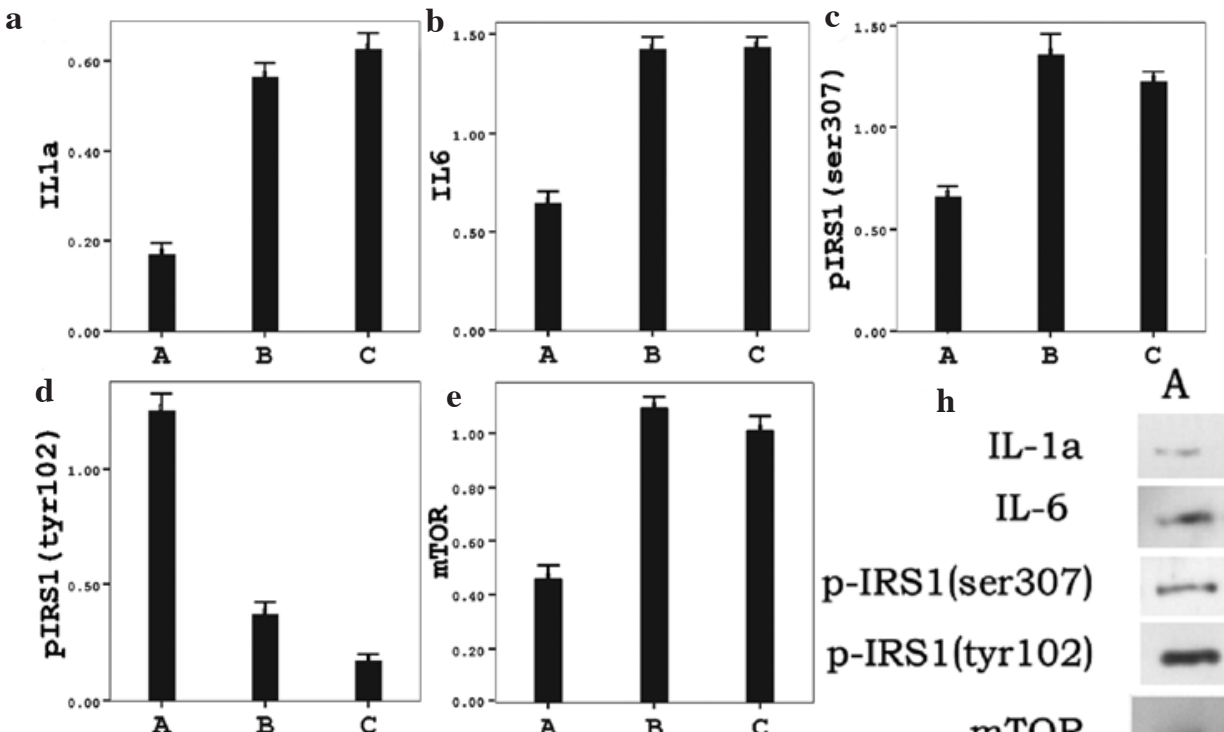

h

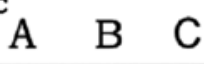

IL-1a

IL-6

p-IRS1(ser307)

p-IRS1(tyr 102)
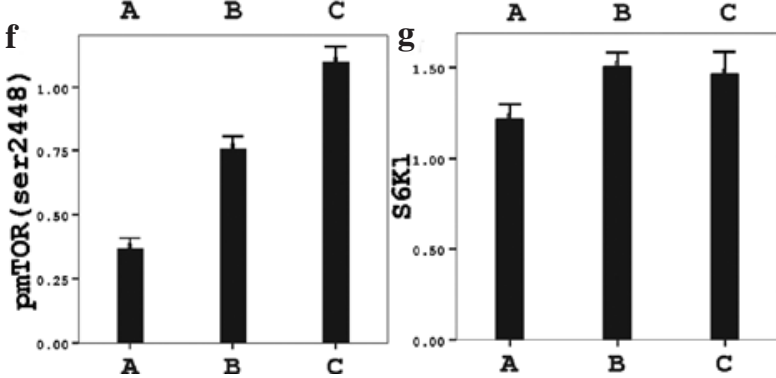

MTOR

p-mTOR(Ser2448)

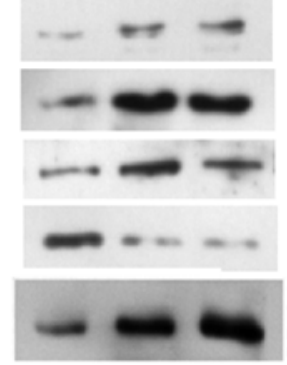

S6K1

GAPDH

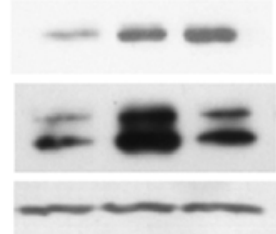

Figure 3. Protein level of IL-1 $\alpha$ (a), IL-6 (b), pIRS-1(Ser307) (c), pIRS-1(Tyr102) (d), mTOR (e) pmTOR(Ser2448) (f) and S6K1 (g). GADPH was used as an internal control. Values are the means \pm SD. A, control group; B, HFD-8 group; C, HFD-16 group. *A compared with B or C, P<0.05. * B compared with C, $\mathrm{P}<0.05$. A representative Western blot image is shown (h).

influence the incidence and development of NAFLD $(10,14)$. Various factors that may affect both IR and chronic liver inflammation appear to play important roles in the development of NAFLD.

Interfered or impaired insulin signaling has been reported to be responsible for IR (15). mTOR has a wide variety of biological functions and is involved in the occurrence of insulin resistance and the transmission of insulin signals $(5,16)$. To assess the role of mTOR in the occurrence and development of NAFLD, SD rats were used as models in this study. The establishment of an SD rat model of NAFLD by feeding the rats a high-fat diet was considered to be successful, as steatotic liver tissues were identified in the rats by means of pathological examination. The metabolic features of this model included IR, fasting hyperinsulinemia and increased serum transaminase. It was found that mTOR mRNA expression tended to increase progressively and significantly with the progression in pathological morphology from simple fatty liver (in the HFD-8 group) to fatty hepatitis (in the HFD-16 group), which suggests the involvement of mTOR in the etiology and development of NAFLD.

IRS-1 protein is crucial for insulin signaling and may cause IR in the peripheral target tissues of insulin, as it is a mediating factor in insulin signaling disorder $(17,18)$. The function of IRS-1 may be regulated either by IRS-1(Ser307) phosphorylation or by the IRS-1 degradation pathway. Following the stimulation of insulin, tyrosine phosphorylation is a prerequisite for IRS- 1 to function as a transducer of signals (i.e., positive regulation). Stimulation by insulin may also lead to IRS-1 serine phosphorylation, which inhibits the signaling function of IRS-1 (i.e., negative regulation). A balance between positive and negative regulation is important in modulating the function of IRS-1. Previous studies have found that IRS-1(Ser307) overphosphorylation may prevent the interconnection between IRS-1 and insulin receptors and, therefore, may decrease IRS-1 tyrosine phosphorylation $(19,20)$. In line with previous findings, the results of this study showed significantly increased pIRS-1(Ser307) and considerably decreased pIRS-1(Tyr102) levels in rat liver tissues when there was a pathomorphological progression from simple fatty liver (the HFD-8 group) to fatty hepatitis (the HFD-16 group). These results suggest that upregulation of IRS-1(Ser307) phosphorylation may induce additional IR by causing an insulin signaling disorder via inhibition of the interconnection between IRS-1 and insulin receptors, decreasing IRS-1(Tyr102) phosphorylation and affecting the function of IRS-1.

mTOR is a downstream component of the IRS/PI3K/Akt signaling pathway and the nutrient signaling pathway in islet cells (21). Recent studies have found the following: growth factor signals may downregulate the activation of PI3K via mTOR and S6K (22); the mid- and downstream signals of the insulin signaling pathway may also induce insulin resistance by inhibiting the upstream signals through a negative feedback regulation mechanism (23); S6K may cause phosphorylation of IRS-1 serine and IR may be induced by decreased Akt phosphorylation due to a disconnection between IRS and insulin 
receptors (24-26). Briaud et al (27) reported that glucose or insulin-like growth factors in islet $\beta$ cells may cause increased IRS Ser/Thr phosphorylation, decreased IRS expression and increased apoptosis of islet $\beta$ cells by chronic activation of mTOR. Overactivation of the mTOR/S6K1 cascade may inhibit insulin signaling, prompt the occurrence and development of IR and cause metabolic disease and type 2 diabetes (28). Since phosphorylation represents the activation of mTOR, and as total mTOR may not fully reflect the activation of the mTOR signaling pathway (29), phosphorylated mTOR was used in this study as a detection index to investigate the association between the activation of the mTOR signaling pathway and NAFLD. The results of this study revealed significantly increased pmTOR(Ser2448) and S6K1 expression with the pathomorphological progression from simple fatty liver (HFD-8 group) to fatty hepatitis (HFD-16 group), suggesting that overactivation of the mTOR cascade in the liver occurs during the development of NAFLD. Moreover, the expression of pIRS-1(Ser307) was significantly positively correlated with that of mTOR, pmTOR and S6K1, while a significantly negative association was observed between the expression of pIRS-1(Tyr102) and that of mTOR, pmTOR and S6K1. This indicates that overactivation of mTOR may induce IR by causing IRS-1(Ser307) overphosphorylation and decreasing IRS-1(Tyr102) phosphorylation via activating S6K1.

TNF $\alpha$, IL-1 and IL-6 may induce IR by interfering with insulin signaling. IRS is a key site at which inflammatory factors cause IR. TNF $\alpha$ is not only involved in inflammatory reactions, but also plays an important role in fat metabolism as an adipocytokine $(30,31)$. TNF $\alpha$ may induce IR by impairing insulin sensitivity via inhibition of PI3K activity, decreasing expression of glucose transporter 4 (GLUT4) and downregulating PPAR- $\alpha$, adiponectin and IRS-1 $(31,32)$. In addition, IL-6 is an important modulator for energy metabolism balance, and may significantly inhibit insulin-dependent activation of AKT and suppress the expression of IRS-1, GLUT4 and PI3K, thereby affecting post-insulin receptor signaling and causing IR (31). IL-6 mRNA is highly expressed in blood and liver tissues in patients with non-alcoholic steatohepatitis (NASH) (33). Blood cell cultures of NAFLD patients have shown that IL-l $\alpha$ production is increased and positively correlated with fatty hepatitis and IR (34). Insulin tolerance tests revealed lower fasting blood glucose and insulin levels and improved insulin sensitivity in IL-1 $\alpha^{-/}$mice compared with wild-type mice (35). Consistent with the results mentioned above, this study found that the expression of IL- $1 \alpha$, IL- 6 and TNFa mRNA was significantly higher in the HFD-8 and HFD-16 groups than that in the control group. The protein expression of IL-1 $\alpha$ and IL- 6 was also markedly increased in the HFD-8 and HFD-16 groups compared with the control group, suggesting the involvement of inflammatory factors in the incidence and development of NAFLD.

One of the most important characteristics of cell signaling is the construction of a complicated signaling network system in which there is crosstalk between various pathways, with convergence or divergence. This is also underpinned by studies of the mTOR singling pathway. Cytokines such as TNF $\alpha$ may activate mTOR through the PI3K-Akt pathway $(36,37)$, and mTOR may also influence the activity of NF- $\mathrm{KB}$ (38). mTOR phosphorylation is necessary for the activation of NF- $\mathrm{\kappa B}$ p65
(39), and the activation of NF- $\mathrm{KB}$ may induce high expression of TNF $\alpha(40,41)$, suggesting a complex interaction between TNF- $\alpha$, NF- $\kappa B$ and mTOR. This interaction is associated with IKK $\beta / T S C$ : in tumor cells and breast cancer with a TSC mutation, activated NF- $\kappa B$ activity may increase mTOR activity, and in esophageal adenocarcinoma, activated IKK $\beta$ may lead to increased expression of S6K, a downstream gene of mTOR (41). Lee et al (42) confirmed a close association between IKK $\beta$ and TSC1 by demonstrating that IKK $\beta$ activated mTORC1 by phosphorylating the $\mathrm{S} 487$ and $\mathrm{S} 511$ sites of TSC1 and inhibiting the activity of TSC1/2.

The results of the present study showed a significant positive correlation between pmTOR(Ser2448) and TNF $\alpha$ mRNA. pIRS-1(Ser307) was also found to be significantly positively related to pmTOR(Ser2448), TNF $\alpha$ mRNA, S6K1 and mTOR and that pIRS-1(Tyr102) was significantly negatively associated with pmTOR(Ser2448), TNF $\alpha$ mRNA, S6K1 and mTOR. This demonstrates that both the mTOR/S6K1 and TNF $\alpha /$ $\mathrm{NF}-\kappa \mathrm{B}$ signaling pathways play a role in the generation of NAFLD. Therefore, the streaming between these pathways apparently has a great significance for the targeted treatment of NAFLD, and warrants further investigation.

Based on the findings of this study, mTOR appears to be an important influential factor in the occurrence and development of NAFLD, as it is involved in the induction of insulin resistance and chronic hepatitis. This study provides an experimental basis for future investigations of the role of mTOR in NAFLD. Regulation of the expression of mTOR in the liver may be a novel treatment option for NAFLD.

\section{References}

1. Angulo P: Nonalcoholic fatty liver disease. N Engl J Med 346: 1221-1231, 2002.

2. Marchesini G, Bugianesi E, Forlani G, et al: Nonalcoholic fatty liver, steatohepatitis, and the metabolic syndrome. Hepatology 37: 917-923, 2003

3. Chitturi S and Farrell GC: Etiopathogenesis of nonalcoholic steatohepatitis. Semin Liver Dis 21: 27-41, 2001.

4. Fingar DC and Blenis J: Target of rapamycin (TOR): an integrator of nutrient and growth factor signals and coordinator of cell growth and cell cycle progression. Oncogene 23: 3151-3171, 2004.

5. Parkington JD, Siebert AP, LeBrasseur NK and Fielding RA: Differential activation of mTOR signaling by contractile activity in skeletal muscle. Am J Physiol Regul Integr Comp Physiol 285: R1086-R1090, 2003.

6. Helliwell SB, Wagner P, Kunz J, Deuter-Reinhard M, Henriquez R and Hall MN: TOR1 and TOR2 are structurally and functionally similar but not identical phosphatidylinositol kinase homologues in yeast. Mol Biol Cell 5: 105-118, 1994.

7. White MF: IRS proteins and the common path to diabetes. Am J Physiol Endocrinol Metab 283: E413-E422, 2002.

8. Jansen PL: Non-alcoholic steatohepatitis. Eur J Gastroenterol Hepatol 16: 1079-1085, 2004.

9. Kallwitz ER, McLachlan A and Cotler SJ: Role of peroxisome proliferator-activated receptors in the pathogenesis and treatment of nonalcoholic fatty liver disease. World J Gastroenterol 14: 22-28, 2008.

10. Day CP and James OF: Steatohepatitis: a tale of two 'hits'? Gastroenterology 114: 842-845, 1998.

11. Adams LA, Angulo $P$ and Lindor KD: Nonalcoholic fatty liver disease. CMAJ 172: 899-905, 2005.

12. Choudhury $\mathbf{J}$ and Sanyal AJ: Insulin resistance in NASH. Front Biosci 10: 1520-1533, 2005.

13. Duvnjak M, Lerotic I, Barsic N, Tomasic V, Virovic Jukic L and Velagic V: Pathogenesis and management issues for non-alcoholic fatty liver disease. World J Gastroenterol 13: 4539-4550, 2007. 
14. Valenti L, Fracanzani AL, Dongiovanni P, et al: Tumor necrosis factor alpha promoter polymorphisms and insulin resistance in nonalcoholic fatty liver disease. Gastroenterology 122: 274-280, 2002.

15. Morino S, Kondo T, Sasaki K, et al: Mild electrical stimulation with heat shock ameliorates insulin resistance via enhanced insulin signaling. PLoS ONE 3: e4068, 2008.

16. Tzatsos A and Kandror KV: Nutrients suppress phosphatidylinositol 3-kinase/Akt signaling via raptor-dependent mTOR-mediated insulin receptor substrate 1 phosphorylation. Mol Cell Biol 26: 63-76, 2006.

17. Greene MW and Garofalo RS: Positive and negative regulatory role of insulin receptor substrate 1 and 2 (IRS-1 and IRS-2) serine/ threonine phosphorylation. Biochemistry 41: 7082-7091, 2002.

18. Ogawa W, Matozaki T and Kasuga M: Role of binding proteins to IRS-1 in insulin signalling. Mol Cell Biochem 182: 13-22, 1998.

19. White MF: The IRS-signalling system: a network of docking proteins that mediate insulin action. Mol Cell Biochem 182: 3-11, 1998.

20. Chen XL, Xia ZF, Ben DF, Wang GQ and Wei D: Role of p38 mitogen-activated protein kinase in lung injury after burn trauma. Shock 19: 475-479, 2003.

21. Hay N and Sonenberg N: Upstream and downstream of mTOR. Genes Dev 18: 1926-1945, 2004.

22. Harrington LS, Findlay GM and Lamb RF: Restraining PI3K: mTOR signalling goes back to the membrane. Trends Biochem Sci 30: 35-42, 2005

23. Boura-Halfon S and Zick Y: Phosphorylation of IRS proteins, insulin action, and insulin resistance. Am J Physiol Endocrinol Metab 296: E581-E591, 2009.

24. Um SH, D'Alessio D and Thomas G: Nutrient overload, insulin resistance, and ribosomal protein S6 kinase 1, S6K1. Cell Metab 3: 393-402, 2006.

25. Um SH, Frigerio F, Watanabe M, et al: Absence of S6K1 protects against age- and diet-induced obesity while enhancing insulin sensitivity. Nature 431: 200-205, 2004.

26. Harrington LS, Findlay GM, Gray A, et al: The TSC1-2 tumor suppressor controls insulin-PI3K signaling via regulation of IRS proteins. J Cell Biol 166: 213-223, 2004

27. Briaud I, Dickson LM, Lingohr MK, McCuaig JF, Lawrence JC and Rhodes CJ: Insulin receptor substrate-2 proteasomal degradation mediated by a mammalian target of rapamycin (mTOR)-induced negative feedback down-regulates protein kinase B-mediated signaling pathway in beta-cells. J Biol Chem 280: 2282-2293, 2005 .

28. Di Paolo S, Teutonico A, Leogrande D, Capobianco C and Schena PF: Chronic inhibition of mammalian target of rapamycin signaling downregulates insulin receptor substrates 1 and 2 and AKT activation: A crossroad between cancer and diabetes? J Am Soc Nephrol 17: 2236-2244, 2006.

29. Shinohara M, Chung YJ, Saji M and Ringel MD: AKT in thyroid tumorigenesis and progression. Endocrinology 148: 942-947, 2007.
30. Polyzos SA, Kountouras J and Zavos C: Nonalcoholic fatty liver disease: the pathogenetic roles of insulin resistance and adipocytokines. Curr Mol Med 9: 299-314, 2009.

31. Tsochatzis EA, Papatheodoridis GV and Archimandritis AJ: Adipokines in nonalcoholic steatohepatitis: from pathogenesis to implications in diagnosis and therapy. Mediators Inflamm 2009: 831670, 2009.

32. Ishizuka K, Usui I, Kanatani Y, et al: Chronic tumor necrosis factor-alpha treatment causes insulin resistance via insulin receptor substrate-1 serine phosphorylation and suppressor of cytokine signaling-3 induction in 3T3-L1 adipocytes. Endocrinology 148: 2994-3003, 2007.

33. Dogru T, Ercin CN, Erdem G, Sonmez A, Tapan S and Tasci I: Increased hepatic and circulating interleukin- 6 levels in human nonalcoholic steatohepatitis. Am J Gastroenterol 103: 3217-3218, 2008.

34. Poniachik J, Csendes A, Diaz JC, et al: Increased production of IL-1alpha and TNF-alpha in lipopolysaccharide-stimulated blood from obese patients with non-alcoholic fatty liver disease. Cytokine 33: 252-257, 2006.

35. Matsuki T, Horai R, Sudo K and Iwakura Y: IL-1 plays an important role in lipid metabolism by regulating insulin levels under physiological conditions. J Exp Med 198: 877-888, 2003.

36. Yen CJ, Izzo JG, Lee DF, et al: Bile acid exposure up-regulates tuberous sclerosis complex $1 /$ mammalian target of rapamycin pathway in Barrett's-associated esophageal adenocarcinoma. Cancer Res 68: 2632-2640, 2008.

37. Djavaheri-Mergny M, Amelotti M, Mathieu J, et al: NF-kappaB activation represses tumor necrosis factor-alpha-induced autophagy. J Biol Chem 281: 30373-30382, 2006.

38. Dan HC, Cooper MJ, Cogswell PC, Duncan JA, Ting JP and Baldwin AS: Akt-dependent regulation of NF- $\{$ kappa\}B is controlled by mTOR and Raptor in association with IKK. Genes Dev 22: 1490-1500, 2008

39. Vinciguerra M, Veyrat-Durebex C, Moukil MA, RubbiaBrandt L, Rohner-Jeanrenaud F and Foti M: PTEN down-regulation by unsaturated fatty acids triggers hepatic steatosis via an NF-kappaBp65/mTOR-dependent mechanism. Gastroenterology 134: 268-280, 2008.

40. Aggarwal BB, Takada Y, Shishodia S, et al: Nuclear transcription factor NF-kappa B: role in biology and medicine. Indian J Exp Biol 42: 341-353, 2004.

41. Schwabe RF and Brenner DA: Mechanisms of liver injury. I. TNF-alpha-induced liver injury: role of IKK, JNK, and ROS pathways. Am J Physiol Gastrointest Liver Physiol 290: G583-G589, 2006.

42. Lee DF, Kuo HP, Chen CT, et al: IKK beta suppression of TSC1 links inflammation and tumor angiogenesis via the mTOR pathway. Cell 130: 440-455, 2007. 